\title{
The modified three ports laparoscopic gynecological surgery - a follow-up study
}

\author{
Wu Shun Felix Wong* \\ School of Women's and Children's Health, The University of New South Wales, NSW, Australia
}

\begin{abstract}
Objective: The objective of this study is to present a personal series of a modified three-ports laparoscopic surgical approach and its outcomes in the surgical treatment of patients with benign gynecological diseases.

Study design: It is a retrospective surgical series of patients performed by the author, which analyzes 509 women treated with a modified three-ports laparoscopic surgical approach for benign gynecological diseases from January 2014 to August 2018 in his private practice in Hong Kong. It is also a follow-up study from a preliminary report of 72 cases in 2014.

Results: This paper presented the patients' characteristics, pathology, outcome, and gynecological procedures performed using a modified laparoscopic surgical approach. The surgical procedures mainly included laparoscopic hysterectomy, adnexal ovarian and tubal surgeries, laparoscopic myomectomy, and laparoscopic adhesiolysis. There was no significant difference in operative complications, blood loss, or length of hospital stay when compared to conventional laparoscopic surgery reported in the literature. The perioperative complications are minimal in this personal series. In this study, laparoscopic myomectomy and laparoscopic adhesiolysis for pelvic endometriosis were found to take a longer time to perform using this approach compared with other procedures.
\end{abstract}

Conclusion: This paper reported a feasible and safe surgical approach in laparoscopic surgery for women with gynecological diseases. The operation time, blood loss, length of hospital stay, and outcomes were comparable to conventional laparoscopic surgery.

\section{Introduction}

Nearly all surgery produces surgical scars. For the time being, no endoscopic surgery is scarless. The new single-incision laparoscopic surgery (SILS) and natural orifice surgical approaches are the latest innovations in surgical endoscopy. They produce reduced numbers of scars that may not be visible to others except for the doctors and the patients. However, these surgical approaches may require the modifications of surgical instruments and a longer learning curve to facilitate the surgery. These laparoscopic approaches help to reduce the number of accessory ports and also their potential complications. Thus, they provide a safe, "scarless" and an esthetic option than conventional laparoscopy or open surgery $[1,2]$. Because there is a single incision, patients often enjoy a speedy recovery after the surgery and are impressed with this single scar approach.

The single port surgical approach, however, is clumsy to perform because of its operating limitations. Even with the use of flexible camera and modified laparoscopic instruments to facilitate the procedures, these additional instrumentations are expensive and also do not overcome the intrinsic difficulties to perform these single port surgeries. This single port approach also requires a specially designed, expensive portal device to enable surgeons to achieve and complete their operations. It imposes additional costs without any documented operative advantages.

Although some surgeons used multiple conventional trocars placed at the umbilicus to perform cosmetically the same, a single-site, multiple incisions laparoscopic surgery (at the umbilicus), they still encountered the same technical difficulties as the single port surgery.
Wong and Lee, in 2014, reported and shared their preliminary experience of a modified three-port laparoscopic surgery technique [3]. Using this "modified 3 ports approach", the author now demonstrates in a larger series of patients that this laparoscopic approach possesses the technical advantages of "reduced scars", esthetic and ease over the conventional laparoscopy.

\section{Surgical approach}

This modified three-port laparoscopic surgery has the crucial features to position the three portal incisions in the abdomen that can hardly be noticeable after their healing. The positions of these three ports are 1) a small 5-mm incision is at the 8 o'clock position of the umbilicus which allows direct entry of an optical tip trocar and cannula as described previously by the author [4]; 2) another 5 -mm trocar/ cannula is at $0.3 \mathrm{~cm}-0.5 \mathrm{~cm}$ away, but at the 4 o'clock position of the umbilicus; 3 ) an additional port is placed at either the mid-suprapubic point just below the level of the pubic hairline or anywhere at the left or right lower abdominal quadrant wherever convenient for the surgeon's operating manipulation. Sometimes, a 3-mm mini-port trocar and

${ }^{\star}$ Correspondence to: Felix Wong, Women's and Baby Clinic, Suite 831, Central Building, 1-3 Pedder Street, Central, Hong Kong, E-mail: fwong3@hotmail.com.hk

Key words: gynecological diseases, modified 3-ports, laparoscopic surgery

Received: June 15, 2020; Accepted: August 18, 2020; Published: August 24, 2020 
cannula were used, which could result in an even smaller sized scar, to enhance the cosmetic effect (Figure 1).

A straight $5 \mathrm{~mm}$ 0-degree conventional laparoscope and standard operative instruments are used with this approach. The use of angulated instruments or flexible camera is not required. Even with conventional instruments and laparoscope, there are only minimal clashing between the laparoscope and the other umbilical portal instrument. Because of the limited space between them, the two cannulas' heads at the umbilicus should be of small-sizes; the laparoscope and the operating instrument can then manipulate freely at operation without any interference.

When it is necessary to retrieve surgical specimens from the peritoneal cavity, one of the $5-\mathrm{mm}$ port incision at the umbilicus is enlarged to a $10-12 \mathrm{~mm}$ incision, followed by the insertion of a blunt $10-12-\mathrm{mm}$ trocar and cannula. This enlarged umbilical port of 11-12 $\mathrm{mm}$ size serves to introduce an endobag for the collection and retrieval of the operative specimen. On the other hand, this enlarged port/wound can be used for inserting a 10-mm grasping forceps or a $12-\mathrm{mm}$ electric morcellator for myomectomy and morcellation. The introduction of a needle for surgical suturing is also feasible with this portal arrangement. The instruments at the left lower abdominal port will be manipulated as with our usual standard three ports or four ports laparoscopic procedure.

In this approach, the surgical steps for the common gynecological operations are similar to those of conventional laparoscopic procedures. Special surgical techniques if any, are described as follows:

\section{Adnexal surgery}

The techniques to perform salpingo-oophorectomy, oophorectomy, and ovarian cystectomy had been previously described in the preliminary paper in 2014. There was no major change in surgical techniques. The adnexal disease was easily exposed by moving the uterine manipulator. The operation proceeded to divide any adhesions, perform ovarian cystectomy or remove pelvic tumors by blunt and sharp dissection using two operating instruments without crossing over, and finally, hemostasis was ensured. An endobag was inserted through a $10 \mathrm{~mm}$ port at the umbilicus; specimens were placed inside the bag and removed via the $10 \mathrm{~mm}$ portal wound at the umbilicus. Larger specimens, including large dermoid cyst or chocolate cyst wall, were retrieved by piecemeal removal within the bag at the umbilicus. The rest of the surgery, such as checking hemostasis, placement of

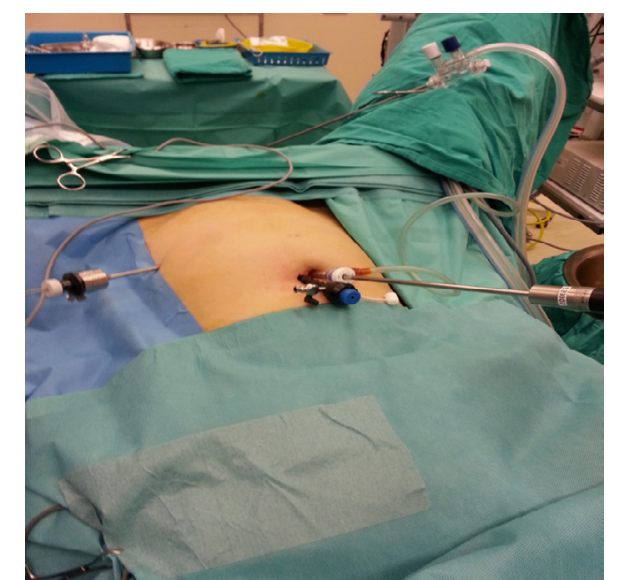

Figure 1. The positons of surgical ports in the "modified 3 ports" laparoscopic surgery - two ports at the umbilicus, either two $5 \mathrm{~mm}$ cannula or one $5 \mathrm{~mm} /$ one $10 \mathrm{~mm}$ cannula. The third trocar and cannula are positioned at the left lower quadrant of the abdomen adhesive barrier materials, surgical suturing, were performed similarly to the conventional laparoscopy.

\section{Myomectomy}

There had not been much technical improvement in dealing with fibroid larger than $8 \mathrm{~cm}$ in diameter because it would be difficult to remove a large fibroid from the uterus. The surgical manipulation at the level of the umbilicus would not allow easy removal of a large fibroid. To place the operative port at a higher abdominal position, like the LeeHuang point, would defeat the purpose of this approach [5]. Therefore laparoscopic myomectomy for a larger fibroid $>8 \mathrm{~cm}$ was performed using other conventional laparoscopy approaches. After extraction of the fibroid from the uterus, the fibroid was then placed in an endobag and left temporarily aside. The myomectomy wound was then sutured using barbed sutures to close the wound and to control hemostasis. The 5-mm laparoscope was then transferred to the lateral abdominal port to provide direct monitoring of the morcellation process, to ensure safety. The fibroid was then morcellated and removed piece by piece using a $12-\mathrm{mm}$ electrical morcellator under direct visualization. There was no surgical failure after those huge fibroids were excluded for this approach. The myomectomy procedure was shown in Figure 2.

In this approach, the lateral lower quadrant abdominal wound remained as a $5 \mathrm{~mm}$ portal wound, which was used to monitor the morcellation procedure, to remove debris and assist in the uterine wound suturing.

\section{Hysterectomy}

Laparoscopy-assisted vaginal hysterectomy $[\mathrm{LAVH} \pm \mathrm{BSO}]$ was the only procedure for patients who underwent a hysterectomy in this series of patients. In the early stage, the author had used total laparoscopic hysterectomy (LH) and laparoscopic subtotal hysterectomy (LSH) and LAVH using this new portal arrangement, LAVH was then chosen. Technically, with this approach, both TLH and LSH could be performed, but the dissection of the vaginal vault and vault suturing was found to be more difficult. With the LAVHs, after the laparoscopic part was completed, the uterus could be easily removed through the vagina with or without morcellation, and the vaginal vault closed. There were no technical difficulties encountered while performing the LAVH procedure with this approach. Therefore, LAVH is the most suitable procedure for using this approach because technically, it is an easier procedure compared to the total laparoscopic hysterectomy, demanding less time, less bleeding, less skill, and laparoscopic suturing.

\section{Result}

Laparoscopic surgery using the modified 3 ports approach was performed in 509 women for various gynecological diseases from 20142018 (Table 1).

Two hundred and thirty-seven patients underwent laparoscopic adnexal surgery, which included operations for endometriomas, benign ovarian cysts, ectopic pregnancy, dermoid cysts, paraovarian cysts, hydrosalpinx, and one ovarian fibroma. One hundred and fifty-six patients had LAVH \pm BSO for fibroids, adenomyosis, DUB, polyps, CIN2-3, and a hematometra, and 86 patients had laparoscopic myomectomy and adenomyomectomy. Thirty patients had laparoscopic adhesiolysis, excision of pelvic endometriosis, and drainage of an abscess. All surgeries were successfully performed using this approach. There is no intraoperative morbidity recorded, and the average hospital stay was 2.7 days (range 2.1 to 3.3 days). Within one week after surgery, there was one case of bowel perforation and one bladder perforation, 
both due to thermal injuries. Nine patients with mild umbilical wound infections were recorded. One patient had her operation converted to laparotomy because of unexpected ovarian malignancy. Overall, cosmesis was excellent with scars hardly visible, as shown in Figure 3.

\section{Discussion}

Minimally invasive surgery has now established as the standard surgical procedure for benign gynecological diseases and plays an increasing role in their treatment. However, the conventional laparoscopic surgery using three to four ports is now considered by some to be unnecessary. Single port surgery is a rapidly developing surgical procedure and marks a new frontier in laparoscopy. It has not widely adopted in gynecological surgery for several reasons: (1) Inconvenient operations with clashing and "sword fighting" of the instruments. (2) Loss of angulation directions for suturing, tissue separation, and hemostasis. (3) Some surgeons may require purposely designed instruments to facilitate the ease of surgery, thus increasing the cost of operation. (4) More extended training because of the long

Table 1. Showed that 509 patients had the modified 3 ports laparoscopic surgery performed from 2014 to 2018 . The patients' characteristics, pathology, gynecologic procedures, and outcomes were listed.

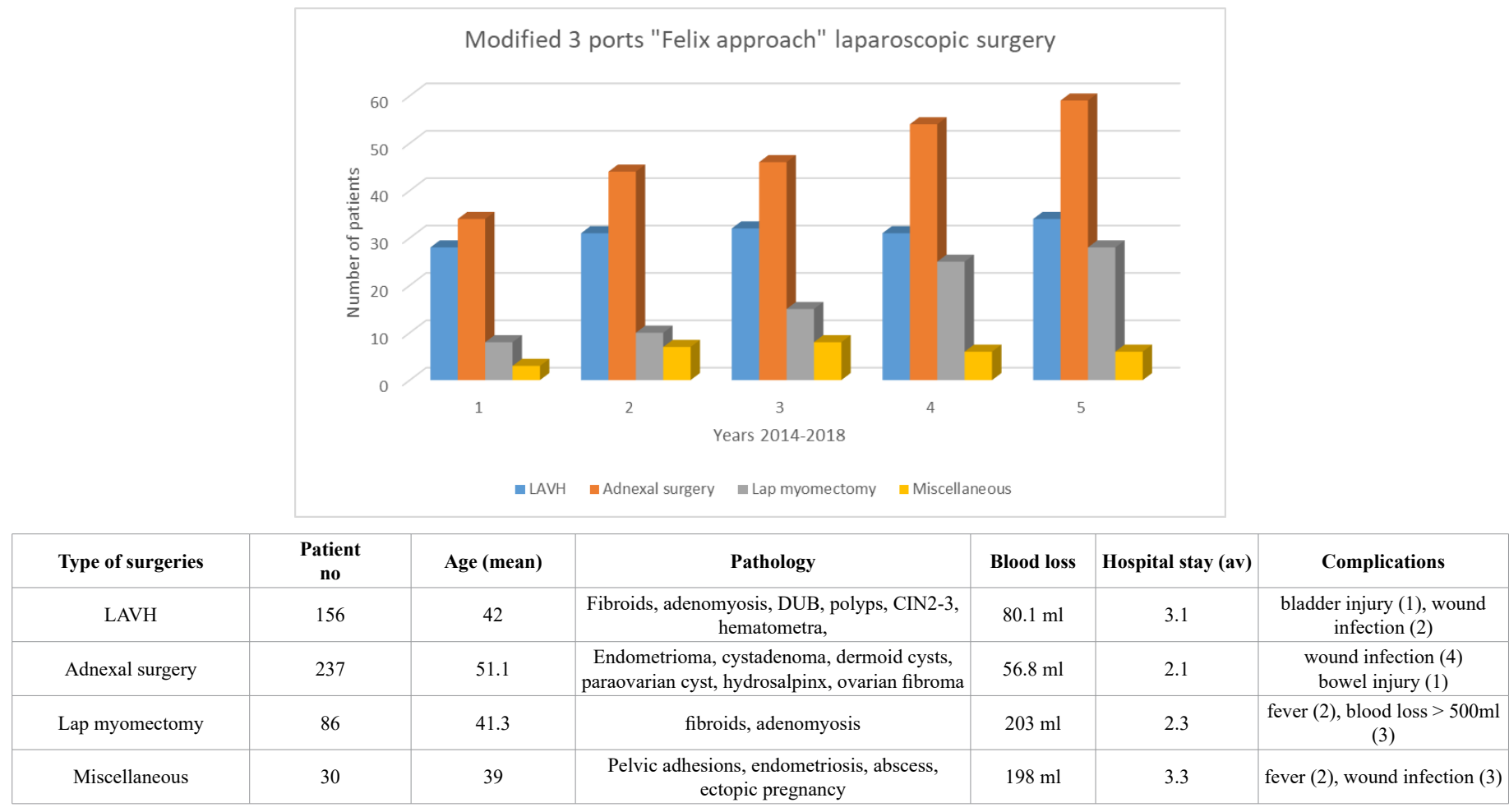

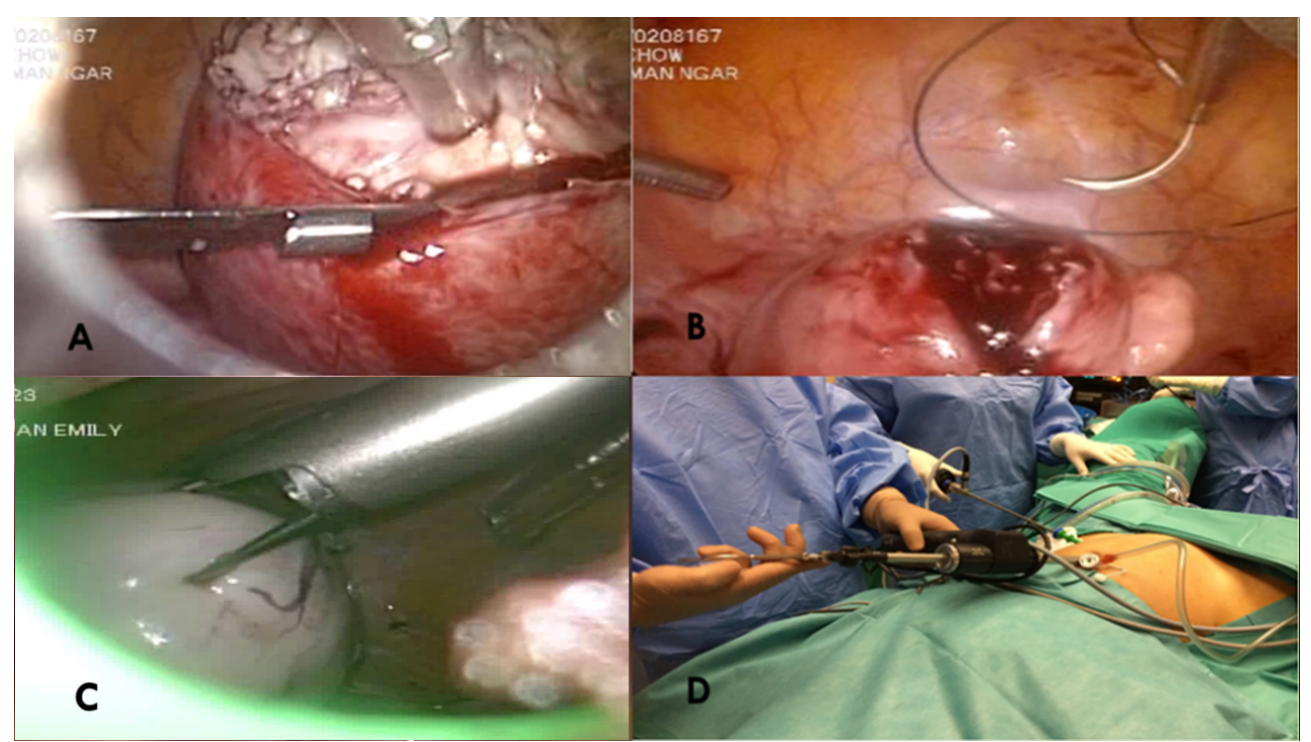

Figure 2. Laparoscopic myomectomy using modified 3 ports approach A) Myomectomy performed in the usual manner. B) Myomectomy wound suturing using barbed suture. C) Electric morcellation of fibroid. D) External view of morcellation of fibroid under direct vision by laparoscopy from the lateral abdominal port 


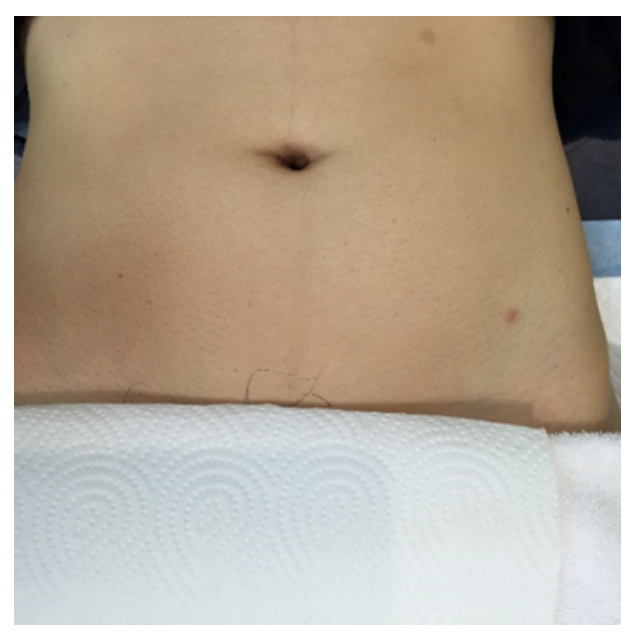

Figure 3. Good aesthetic scars after the modified 3 ports laparoscopic surgery

learning curve. (5) More demanding skills in challenging situations. Despite its reported success in some centers, short-term or long-term complications still occur [6].

This present approach has adopted the single-port laparoscopic surgery concept of reduced scars to modify the three-port surgical approach, with two ports placed at the umbilicus, and one port elsewhere in the body. Any laparoscopic procedures for benign gynecological diseases can be performed with the surgeon operating with the common laparoscopic instruments, manipulating at portal sites similar to conventional laparoscopic surgeries. This approach will not only shorten the learning curve and reduce the difficulties encountered in single port surgery but will also provide satisfactory outcomes.

With the increasing number of patients operated in this personal series, there are additional techniques that may need elaboration to make it easier for others to follow and perform laparoscopic surgery with this approach. The following is a list of advice in addition to those presented in the preliminary study [3].

(1) A $5 \mathrm{~mm}$ laparoscope instead of a $10 \mathrm{~mm}$ laparoscope should be used. However, it can pose some difficulties in maintaining a consistently good visual field because it is easy to get obscured by blood contamination or smoke, resulting in blurred vision. When this happens, clear vision can be regained by touching the tip of the laparoscope to the surrounding bowel or pelvic peritoneum. Time may be saved by not having to remove the laparoscope for cleaning.

(2) The laparoscope should be held behind and view the operating instrument at a distance to avoid clashing with operating instruments during an operation.

(3) Due to the small caliber of the $5 \mathrm{~mm}$ laparoscope lens, the use of an older laparoscope, impaired light cord, any increased bleeding in the pelvis will cause a dark and unclear operating field. The laparoscope and light cord should be well maintained and checked before the operation. Bleeding should be controlled promptly, and hemostasis achieved to allow a clear view for easy operation.

(3) The clashing of the laparoscope with the operating instrument at the umbilicus can still be a problem. The movements of both should be in the same direction together, not to crossing each other. However, experience in this approach is essential to perform the surgery smoothly and safely.
(4) Fibroids about $8 \mathrm{~cm}$ or less in size can be satisfactory and conveniently morcellated inside an endobag under direct laparoscopic vision or morcellated by a cold knife at the umbilical wound by enlarging the two umbilical portal wounds. The latter approach may, however, create a bigger umbilical scar.

(5) The use of barbed sutures, e.g., V-lock suture, will make it easier to close the myomectomy wound. However, the risks of barbed sutures should be used with caution because complications arising from this suture had been reported [7].

(6) Complicated surgeries like severe pelvic adhesions and advanced pelvic endometriosis are still difficult to perform with this approach because of the extent of disease can be widespread, which could be away from the operation field of the laparoscopic instruments.

(7) On some occasions, it is not a shame to abandon this approach, make a few more portal incisions, and operate using three to four ports. Alternatively, a wound can be enlarged to make the procedure operable and safe.

(8) If there are suspected adhesions at the umbilical site after previous laparoscopic surgeries, the introduction of the laparoscope should be under laparoscopic vision using an optical guided trocar, e.g., the Endopath Xcel Trocar, with a technique previously described by the author [4]. The direction of the trocar entry should be directed to the side less likely to have adhesions such as left-sided entry when previous surgery was on the right side. Most importantly, any suspected injury can be recognized, and repair is done promptly.

In this larger follow-up series, some limitations of the study mentioned previously, such as single-surgeon experience, noncomparative study design, and patient selection bias, are less of a problem. Due to the difficulties of randomized studies in a single surgeon operation situation, surgical techniques can be biased and limited toward one's experience, for example, the LAVH approach for hysterectomy in this study. Nevertheless, the outcome of this laparoscopic approach is obvious with the following advantages; (1) less clashing of instruments, operations are mainly performed in a way similar to the conventional laparoscopic surgery; (2) a less expensive and a more cost-effective procedure by using standard instruments and laparoscopy; (3) it offers a familiar view of the operation and manipulative movement of the instruments same as conventional laparoscopy. Because its technical difficulty stands between conventional laparoscopy and single-port surgery, the learning curve will be shorter for those who are interested in adopting this approach.

\section{Ethic statement}

The author declares that all data were collected meeting ethical guidelines.

\section{Disclosure statement}

The author has no conflicts of interest.

\section{Funding sources}

There are no funding sources.

\section{References}

1. Fader AN, Rojas-Espaillat L, Ibeanu O, Grumbine FC, Escobar PF (2010) Laparoendoscopic single-site surgery (LESS) in gynecology: a multi-institutional evaluation. Am J Obstet Gynecol 203: 501. [Crossref] 
2. Behnia-Willison F, Foroughinia L, Sina M, McChesney P (2012) Single incision laparoscopic surgery (SILS) in gynaecology: Feasibility and operative outcomes. Aust N Z J Obstet Gynaecol 52: 366-370. [Crossref]

3. Wong FWS, Lee ETC (2014) A modified three-port "hidden scars" surgical approach in gynecology: A surgeon's experience of an initial 72 cases. Gynecol Minim Invasive Ther 3: 43-46.

4. Wong WSF (2013) A safe optically guided entry technique using Endopath Xce Trocars in laparoscopic surgery: A personal series of 821 patients. Gynecol Minim Invasive Ther 2: 30-33.
5. Lee CL, Huang KG, Jain S, Wang CJ, Yen CF, et al. (2001) A new portal for gynecologic laparoscopy. Gynecol Minim Invasive Ther 8: 147-150.

6. Park JY, Kim TJ, Kang HJ, Lee YY, Choi CH, et al. (2013) Laparoendoscopic single site (LESS) surgery in benign gynecology: perioperative and late complications of 515 cases. Eur J Obstet Gynecol Reprod Biol 167: 215-218.

7. Lee ETC, Wong FWS (2015) Small bowel obstruction from barbed suture following laparoscopic myomectomy-A case report. Int J Surg Case Rep 16: 146-149. [Crossref]

Copyright: $(2020$ Felix Wong WS. This is an open-access article distributed under the terms of the Creative Commons Attribution License, which permits unrestricted use, distribution, and reproduction in any medium, provided the original author and source are credited. 